\title{
High-resolution manometry and impedance-pH/manometry: novel techniques for the advancement of knowledge on esophageal function and their clinical role
}

\author{
C. Ciriza-de-los-Ríos and F. Canga-Rodríguez-Valcárcel \\ Service of Digestive Diseases. Hospital 12 de Octubre. Madrid, Spain
}

Ciriza-de-los-Ríos C, Canga-Rodríguez-Valcárcel F. High-resolution manometry and impedance-pH/manometry: novel techniques for the advancement of knowledge on esophageal function and their clinical role. Rev Esp Enferm Dig 2009; 101: 861-869.

\section{INTRODUCTION}

The study of esophageal function is based on the study of esophageal motor activity using conventional esophageal manometry (CEM), which has proven useful in the diagnosis of patients with functional dysphagia and other esophageal motor disorders (1). Most consensus guidelines recommend contractile activity monitorization with 4 to 8 sensors, including a 6-cm "e-sleeve" to minimize displacement at the lower esophageal sphincter (LES) (2). In the last few years new techniques are allowing a better understanding of esophageal motor disorders. These include high-resolution manometry (HRM) and impedance combined with manometry. Both are relevant research tools that are being increasingly implemented in daily clinical practice (3).

The study of pathological acid reflux as a potential cause for most motor disorders and symptoms as experienced by patients with functional esophageal disease is undertaken with a 24-hour ambulatory $\mathrm{pH}$-metry with one or several recording sites, and is still considered the gold standard for the study of non-erosive gastro-

Received: 31-05-09.

Accepted: 05-06-09.

Correspondence: C. Ciriza de los Ríos. Servicio de Enfermedades Digestivas. Hospital Universitario 12 de Octubre. Avda. de Córdoba, s/n. 28041 Madrid.e-mail: constanzacarpa@gmail.com esophageal reflux disease (GERD). However, there is a growing interest in the diagnosis of patients with atypical reflux complaints and of those refractory to therapy with proton-pump inhibitors (PPIs), where $\mathrm{pH}$-metry plus impedance plays a relevant role given its ability to detect weakly acidic or non-acidic reflux episodes (4).

\section{THE STUDY OF ESOPHAGEAL MOTOR FUNCTION}

High-resolution manometry

\section{HRM basics and benefits}

HRM allows more accurate measurements of pressure changes along the esophagus as a result of a number of complex physiological situations, and the analysis and plotting of these changes for clinical use represents a huge challenge. A brief description of these physiological changes follows.

The upper esophageal sphincter (UES) and proximal esophagus are areas where striated muscle predominates, hence they contract much more rapidly than the distal esophagus and lower esophageal sphincter (LES), where smooth muscle predominates. Not only does this occur first but also the UES and esophagus move cephalad with deglutition because of the contraction of pharyngeal muscles and a shortening of longitudinal muscle fibers during peristalsis. Finally, the UES and LES have an asymmetric radial morphology, which in the former case results from its anatomy and in the latter case from contraction as induced by the crural hiatus.

All these physiological events induce various changes that are difficult to interpret with conventional manometry (a water perfusion system). In order not to underestimate such changes a highly sensitive, versatile technology is needed, which HRM can offer. HRM detects 
pressure or morphology changes more accurately because of novel measurement devices, primarily an electronic sleeve and solid-state transducers that can adapt themselves to any areas, and can be grouped together based on needs and local characteristics, which largely addresses prior concerns as will be discussed below.

HRM is not in itself a novel technique for the study of esophageal motility, but represents a modified pre-extant modality. Early developmental research was carried out by Clouse and Staiano (5) in 1991 in gastrointestinal motility research laboratories. What is novel about HRM versus previous digestive motility-related diagnostic systems is the use of a probe incorporating 36 solid-state pressure sensors with a spaced at 1-cm intervals (Sierra Scientific Instruments, Inc., Los Angeles, CA). Software was also developed that includes algorithms to process an enormous amount of manometric data, as well as pressure topographic plots to allow a better understanding of said data (6). Topographic pressure plots render sphincter areas - as well as their location and measurements, including morphology, length and pressures - much more easily recognizable. Also the dynamic behavior of these structures is analyzed with greater diagnostic precision, unaffected by their relaxation-related displacement, which is largely due to the software's ability to implement an electronic sleeve (e-sleeve) for the analysis of LES, which warrants higher-stability measurements (7).

All these innovations facilitate an understanding of esophageal functional anatomy with a rapid, easier reading of manometric data. In this respect a study compared CEM results (4 sensors and stationary pull-through manometry) to HRM results by enrolling 212 patients with various indications for esophageal manometry (8). Similar diagnostic criteria were used for both techniques, and most cases $(88.2 \%)$ showed diagnostic consistency; however, CEM could not detect 6 of 36 achalasias, all of them successfully identified by HRM, nor 12 patients with incomplete LES relaxation, again properly detected with HRM. Therefore, this study concluded that HRM was more sensitive than CEM for the study of esophageal motor disorders.

HRM is also superior in assessing UES dynamics and the functional anatomy of the gastroesophageal junction (GEJ), and allows peristaltic contraction segmentarity to be analyzed $(5,6,9)$. Thus, esophageal topographic analyses using HRM have shown that bolus progression along the esophageal body is uneven, and characterized by a sequence of contractile events along four pressure segments. The first segment represents the striated-muscle component of the proximal esophagus, and extends from UES to the first esophageal pressure drop at the aortic arch area. This low-pressure segment represents the transition zone. Another area of interest corresponds to the lower two thirds of the esophagus, where smooth muscle predominates, which may in turn be divided into two overlapping neuromuscular segments. The fourth contractile segment is the GEJ (10) (Fig. 1). These functional

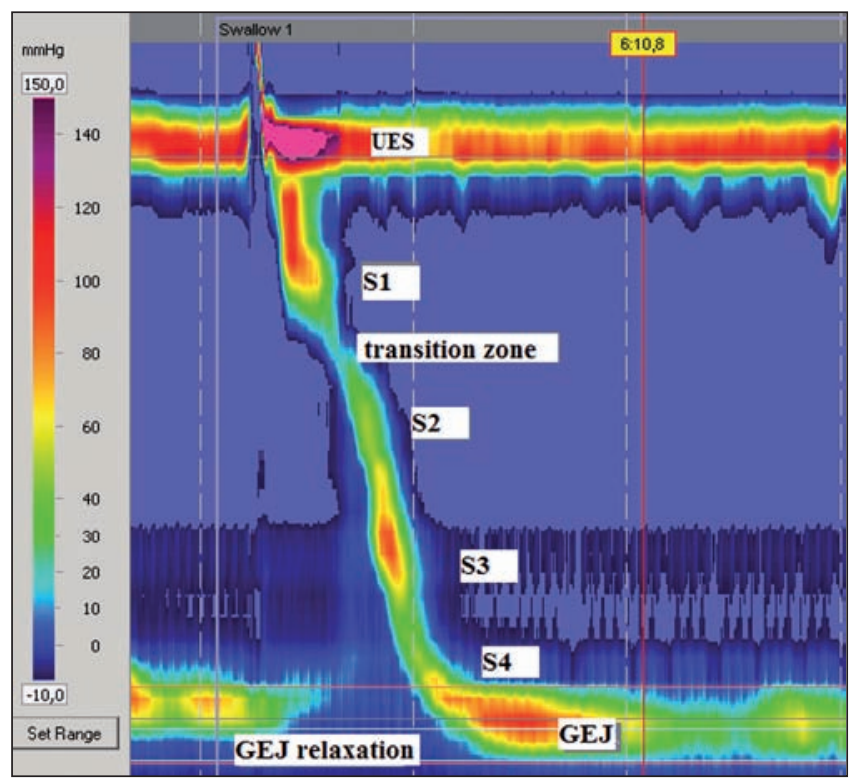

Fig. 1. Normal peristaltic sequence. EES: upper esophageal sphincter UGE: gastro-esophageal junction. S1 is the first contractile segment representing the skeletal muscle component of the proximal esophagus, and extending from EES to the first esophageal pressure fall at the aortic arch region. This low-pressure segment represents the transition zone. The area corresponding to the lower two esophageal thirds is considered an area with predominant smooth muscle that may be further divided up into two overlapping neuromuscular segments (S2 and S3). The forth contractile segment (S4) represents the LES.

segments with differing dynamic components are better assessed by HRM. Thus, this technique allows us to understand the esophageal transition zone not only as a decrease in peristaltic pressure amplitude but also as a transition between contractions that propagate on absolutely different physiological actions - at the proximal, mainly striated-muscle segment they are attributed to a sequential activation of brain motor neurons, whereas at the distal, mainly smooth-muscle segment they would result from a balance between inhibitory and excitatory interneurons at the myenteric plexus (11). This improved functional understanding of the transition zone leads us to deduct that an abnormal delay between the end of the proximal contraction and the beginning of the distal contraction, or an interruption between two contractions, may lead to dysphagia $(7,10,12)$. Another area where HRM proves superior is in establishing an accurate representation of the relationship between contractile wave closure force (contractile pressure), esophageal clearance force (intrabolus pressure) and esophageal outflow resistance (pressure gradient accross GEJ). Esophageal peristalsis pattern and sphincter activity define whether esophageal motor activity is normal or abnormal. Esophageal intrabolus pressure and pressure gradient accross GEJ define whether or not this activity is consistent with effective function (13). 
Thus, HRM represents an advance over CEM as it is not restricted to pressure measurements but also assess esophageal function by establishing an objective link between pressure measurements and esophageal bolus progression. This is significant in that changed bolus progression is more readily correlated to esophageal symptoms. The CEM-HRM comparison shows that the latter is more accurate in predicting bolus transit changes as measured with videofluoroscopy, particularly in patients with mild to moderate esophageal dysfunction. Another relevant feature of HRM is improved focal dysmotility identification, which corroborates the fact that functionally relevant motor changes may be limited to short esophageal segments, and hence may escape detection by sensors wide apart (7) (Fig. 2).

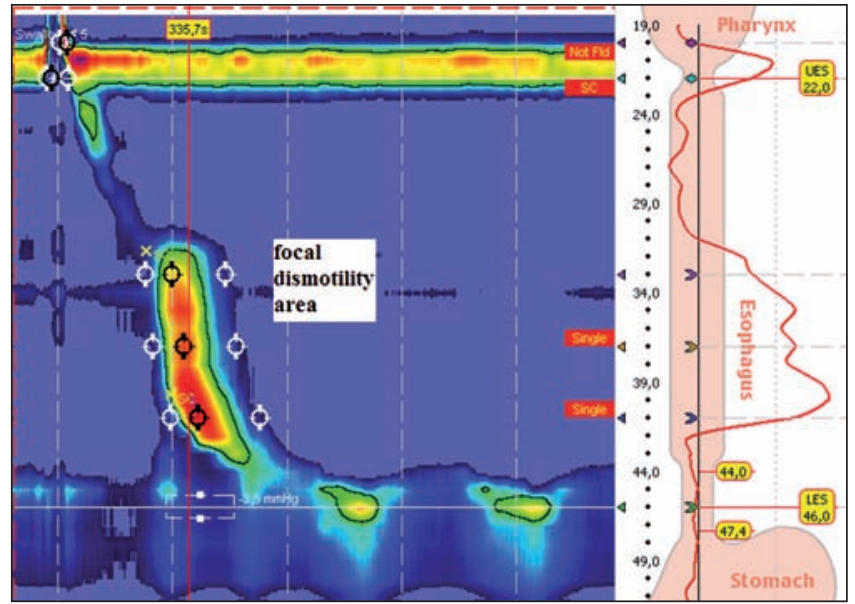

Fig. 2. A focal dysmotility area in a patient with dysphagia. An aperistaltic segment is seen at the mid esophageal third (segmentary spasm) with a loss of coordination between the proximal esophagus and the mid-distal esophagus.

\section{HRM in clinical practice}

HRM represents an improvement over CEM as commonly used for decades, and it seems only logical to believe that HRM will ultimately replace CEM in routine clinical practice. HRM methodology is easier as both sphincters are simultaneously identified with no need for the initial pull-trough protocol for LES localization (10). Other than this the procedure is similar for both techniques, with 10 fluid swallows at 30-second intervals.

The increasingly common use of esophageal topographical analysis makes it advisable to reconsider the esophageal motility categories used by CEM, which have no regard for the new data HRM provides. A parallel classification with improved quality by including HRM's most relevant findings would be an option. In this respect a new esophageal motility classification has been suggested based on the results obtained from 75 control subjects and their extension to 400 patients (14) (Table I).
Table I. Esophageal motility criteria using HRM

\begin{tabular}{ll}
\hline & Cases (\%) \\
\hline Normal & $91(23 \%)$ \\
- $\mathrm{PFV}<8 \mathrm{~cm} / \mathrm{s}$ in $>90 \%$ of deglutitions & \\
- $\mathrm{DCl}<5000 \mathrm{mmHg}$ & \\
$\quad$ deglutition (eSleeve $3-\mathrm{G}$ nadir $<15 \mathrm{mmHg})$ &
\end{tabular}

Peristaltic dysfunction

- Mild: 3-6 deglutitions with failed peristalsis or a defect $>2 \mathrm{~cm}$ in isobaric contour, $30 \mathrm{mmHg}$ of distal esophageal peristalsis (15 mmHg in proximal-mid esophagus)

- Severe: $>7$ deglutitions with failed peristalsis or a defect $>2 \mathrm{~cm}$ in the isobaric contour, $30 \mathrm{mmHg}$ of distal esophageal peristalsis

Aperistalsis $29(7.3 \%)$

- Absence of sustained pressure above a 30-mmHg isobaric contour at the distal esophageal segment in any deglutition

- Scleroderma pattern: absence of sustained pressure above a 30-mmHg isobaric contour at the distal esophageal segment in any deglutition, and mean LES pressure $<10 \mathrm{mmHg}$

Hypertensive peristalsis $37(9.3 \%)$

- $\mathrm{PFV}<8 \mathrm{~cm} / \mathrm{s}$ in $>90 \%$ of deglutitions

- Mean DCl > 5000 mmHg

- Symptomatic peristalsis ("nutcracker"): mean DCl > 5000 and $<8000 \mathrm{mmHg}$

- Segmentary nutcracker: mean $\mathrm{DCl}>5000 \mathrm{mmHg}$ with only one hypertensive contraction segment $(>180 \mathrm{mmHg}$ )

- Spastic nutcracker: mean DCl >8000 mmHg

- Nutcracker LES: mean DCI >5000 mmHg with hypertensive contraction site (> $180 \mathrm{mmHg}$ ) restricted to LES after contraction

\begin{tabular}{|c|c|}
\hline $\begin{array}{l}\text { Rapidly propagated pressurization } \\
\text { - PFV }>8 \mathrm{~cm} / \mathrm{s} \text { in }>20 \% \text { of deglutitions } \\
\text { - Spasm (increased PFV attributable to rapid contraction wave) } \\
\text { - Compartmentalized pressurization (increased PFV attributable tc } \\
\text { compartmentalized distal esophageal pressurization) }\end{array}$ & $10(2.5 \%)$ \\
\hline $\begin{array}{l}\text { Abnormal LES tonus (minimal expiratory or end-expiratory) } \\
\text { - Hypotensive: mean < } 10 \mathrm{mmHg} \text { with normal peristalsis and } \\
\text { gastro-esophageal junction relaxation } \\
\text { - Hypertensive: mean > } 35 \mathrm{mmHg} \text { with normal peristalsis and } \\
\text { gastro-esophageal junction relaxation }\end{array}$ & 39 (9.9\%) \\
\hline $\begin{array}{l}\text { Achalasia } \\
\text { - Impaired GEJ relaxation with deglutition } \\
\text { - Aperistalsis } \\
\text { - Classic: panesophageal aperistalsis or pressurization with no } \\
\text { - detectable contractile activity in any segment with deglutition } \\
\text { - Vigorous: with distal spasm }\end{array}$ & 73 (18.4\%) \\
\hline $\begin{array}{l}\text { Functional GEJ obstruction } \\
\text { - Impaired GEJ relaxation with deglutition } \\
\text { - Mild: } \mathrm{PFV}<8 \mathrm{mmHg} \text { in }>90 \% \text { of deglutitions with mild } \\
\text { ( } 15-30 \mathrm{mmHg} \text { ) increase in distal esophageal pressurization } \\
\text { - Severe: PFV > } 8 \mathrm{mmHg} \text { in > 20\% of deglutitions with } \\
\text { compartmentalized pressurization }\end{array}$ & $44(11.1 \%)$ \\
\hline
\end{tabular}

This classification relies on the results of HRM studies focusing on sphincter function and the esophageal body, as in CEM but including new parameters to better measure GEJ relaxation, as well as peristaltic contraction integrity and force more accurately and in greater detail.

To this end the definition of GEJ relaxation was modified, and relaxation pressure is quantified over the entire 
deglutition period, as bolus passage through GEJ is not immediate but may take 4 to 5 seconds depending on swallowed volume. Hence, relaxation pressure is quantified by measuring integrated relaxation pressure (IRP) over 4 seconds, which represents the lowest pressure levels during 4 seconds for the period taken by the deglutitional contraction to go through GEJ $(9,15)$.

Bolus transportation will depend on the interaction between GEJ resistance, intrabolus pressure, and post-bolus closure pressure. Therefore, a second part of the analysis entails a definition of peristalsis integrity. The efficacy of distal esophageal emptying is inversely related to peristaltic amplitude in such a way that emptying becomes increasingly impaired with a contraction pressure $<30$ $\mathrm{mmHg}(2,16)$. Establishing an effective contraction pressure $(>30 \mathrm{mmHg})$ is easier when using an isobaric contour, a line delimiting a pressure domain area that includes all pressure values above $30 \mathrm{mmHg}$. A $30-\mathrm{mmHg}$ isobaric contour allows an estimation of pressurization or contraction front velocity ( $P F V$ or $C F V$ ) by measuring pressure from the lower transition zone margin to the upper border of GEJ, and estimating the slope between both (17). Normal deglutition should exhibit an intact isobaric contour at $30 \mathrm{mmHg}$ with a PFV below $7.5 \mathrm{~cm} / \mathrm{s}$ (14). Another HRM parameter is distal contractile integral $(D C I)$, which quantifies the length, force, and persistence of the distal contractile wave. This index is estimated by multiplying the distance between the transition zone and proximal LES border, the time of contraction duration and the integrated pressure amplitude, and the result is expressed as mmHg.s.cm. A DCI > $5000 \mathrm{mmHg} . s . c m$, which represents the $95 \%$ percentile of 75 asymptomatic volunteers, is considered abnormal (14) (Fig. 3).

HRM allows the study of intrabolus pressure, a major marker of the forces opposing peristaltic movement and the occurrence of ineffective bolus progression. In a study of patients with GERD both before and after fundoplication impaired GEJ relaxation was seen to require a higher intrabolus pressure for adequate transit (18).

Another relevant aspect is that HRM allows to readily identify hiatal hernia, which may be a risk factor for GERD, and thus discrimination whether this parameter is present is also of clinical relevance. Prolonged esophageal pressure studies in patients with mild hiatal hernia have shown that the distance between the LES and diaphragm is unstable and varies over time (19). A sliding hernia may be monitored in real time so that it was observed that it can be intermittently reduced for significant time periods. During periods when hernia is obvious the prevalence of gastroesophageal reflux duplicates.

Major differences between the classification suggested by the Chicago team for HRM and prior CEM categories are based on the achalasia subclassification and the functional GEJ obstruction category. A diagnosis with achalasia implies aperistalsis and impaired GEJ relaxation. HRM has identified three specific subtypes that may predict clinical outcome (20). Functional GEJ obstruction

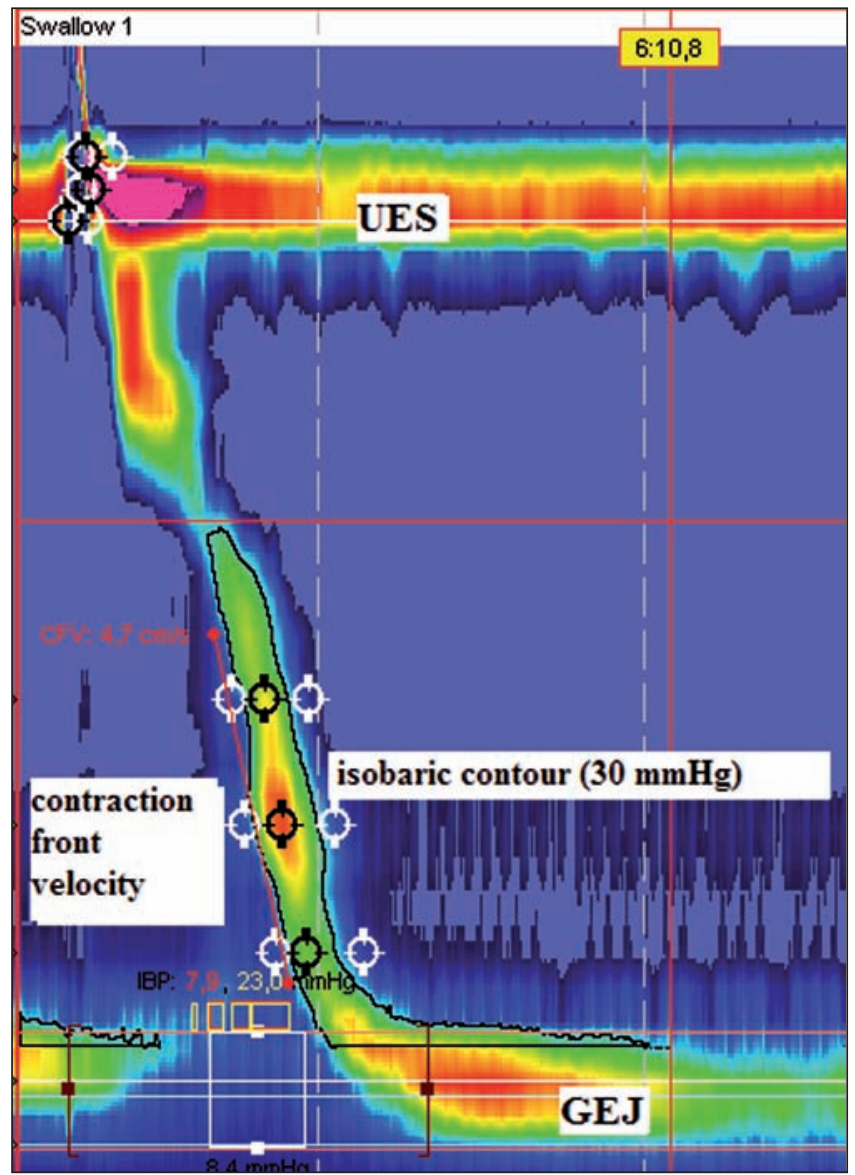

Fig. 3. New parameters in high-resolution manometry. An isobaric contour may be seen, which is the black line delimiting the pressure domain made up of all pressures above $30 \mathrm{mmHg}$. Isobaric contours allow an estimation of pressurization front velocity or contraction front velocity (PFV or CFV) by measuring pressures from the lower margin of the transition zone to the upper margin of the gastro-esophageal junction, and estimating the corresponding slope (red line).

with elevated intrabolus pressure and impaired GEJ relaxation has been seen in patients with fundoplication, esophageal stenosis, and eosinophilic esophagitis, which could not have been adequately defined by conventional manometry $(10,14)$.

High-definition manometry is the most advanced technological development in the field. It allows complete recordings of pressures along the inner esophageal surface, and provides ultra-high resolution for measurements in both directions, axial and circumferential, which permits 3-D moving images. Initial studies suggest that this technique may more accurately study complex anatomical areas such as GEJ, particularly the movement, location and magnitude of the crural diaphragm (2).

In conclusion, HRM is an easier, faster, and more sensitive technique for the study of esophageal motility, and allows a better understanding of esophageal function. The new data provided by HRM demand a new 
esophageal motility classification that still lacks consensus. In the light of the available data HRM seems to provide a higher diagnostic yield in the study of esophageal motor disorders, but this will likely be elucidated in the upcoming future. For the first time esophageal manometry has the potential of entailing not only a major tool for research but also of becoming a standard, objective clinical test.

\section{Impedance combined with manometry}

While HRM is more accurate in predicting impaired bolus transit when compared to videofluoroscopy and conventional manometry, the predictive value of manometry for bolus transit is suboptimal. Esophageal contractions with an amplitude above $30 \mathrm{mmHg}$ have been seen to be usually associated with complete bolus transit (16).

Videofluoscopy, barium studies, and scintigraphy entail patient exposure to radiation. Intraluminal esophageal impedance is a novel technique to measure bolus progression, and can thus be used to quantify bolus transit effectiveness following deglutition (21). Impedance may also be used for the study of gastroesophageal reflux, supplementing the information provided by esophageal $\mathrm{pH}$-metry.

\section{Basics and technique of impedance-manometry}

Impedance is defined as the physical resistance of a given medium to the passage of alternating current. The alternating current circuit is established between two electrodes separated by a non-conductive catheter on which said electrodes are inserted. Impedance may be considered the opposite of electrical conductivity in the medium surrounding both electrodes. Its value, measured out in ohms, will depend on the medium's physico-chemical characteristics (liquid, solid, gaseous, ionization), and on the contact surfaces between electrodes and substances (22).

Air, fluid, and the esophageal mucosa have all unique impedance features, which allow us to discriminate the material between each pair of electrodes. Air is highly resistant to current and shows a high impedance signal, whereas fluid has a low resistance and a low impedance value. The esophageal mucosa has an intermediate impedance, and thus serves as baseline during monitoring. Impedance is highly sensitive to small fluid and gas volumes within the esophageal lumen, and similar impedance drops may be seen with fluid boluses of 1 and 10 $\mathrm{mL}$ (23). This is why degutted or refluxed bolus volume cannot be quantified using impedance (3).

By using several electrodes inserted in the catheter, and defining impedance changes at adjacent pairs of electrodes, one can be stablish the direction of bolus transit within the esophagus and also determine whether complete bolus clearance has occurred $(23,24)$ (Fig. 4).

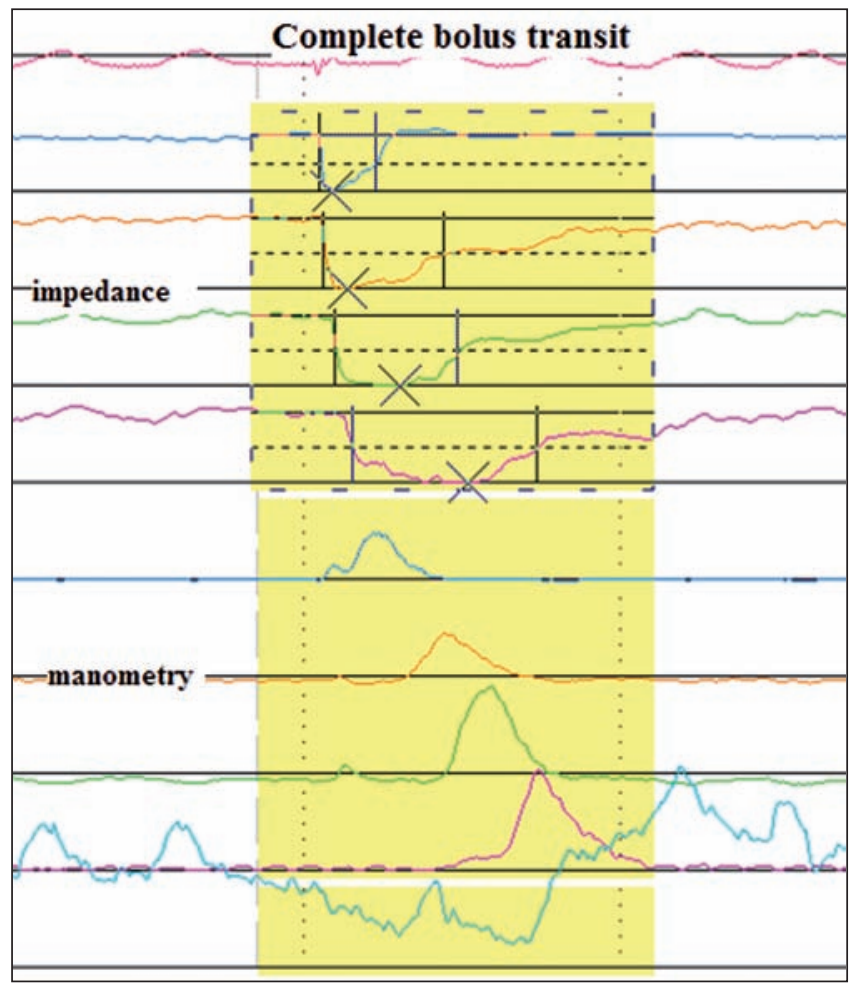

Fig. 4. Impedance-manometry. Impedance channels show a complete bolus follow-through, and vertical bars indicate the bolus coming in and out every impedance segment. Manometry channels reveal a peristaltic contraction.

Available catheters combine manometry with microtransducers (solid-state) and impedance. Ten fluid swallows are usually performed, preferably with saline, which has a higher impedance, at 30-second intervals. Viscous swallows are also usually added, which record more bolus transit failures even in healthy volunteers (25).

Bolus entry into each impedance segment is defined as a $50 \%$ drop in impedance at the recording site, whereas a $50 \%$ increase towards the baseline is correlated with bolus exit during videofluoroscopy (26).

Parameters estimated to assess bolus transit include: a) total transit time (between bolus entry at $20 \mathrm{~cm}$ above LES and bolus exit at $5 \mathrm{~cm}$ above LES); b) bolus presence time (the interval between bolus entry and exit at each impedance recording site); and c) segmental transit times (the interval between bolus entry at a given level above LES and bolus exit at the next most distal level) (3).

\section{Impedance-manometry in clinical practice}

For impedance-manometry to become clinically useful in the study of esophageal motor disorders, it should provide reliable information on bolus transit along the esophagus, and potential impedance-related abnormali- 
ties should explain patient's symptoms in cases where the should manometry fails to do so.

Studies combining impedance to videofluoroscopy have validated the accuracy of impedance in establishing bolus transit. A study in healthy controls found a strong correlation between fluoroscopy and impedance measurements for esophageal filling time $\left(\mathrm{r}^{2}=0.89 ; \mathrm{p}<\right.$ $0.0001)$ and esophageal emptying time $\left(\mathrm{r}^{2}=0.79 ; \mathrm{p}<\right.$ 0.0001 ) (26). Another study found $97 \%$ consistency between the results from both techniques (21). These studies support the predictive value of impedance for bolus transit, at least in healthy subjects.

Normal values for this technique were obtained in a multicenter study including 43 healthy volunteers who performed 10 liquid and 10 viscous swallows. Complete bolus transit was defined when bolus exit was detected in the impendance channels at 15,10 , and $5 \mathrm{~cm}$ above the LES, and incomplete transit when bolus retention was identified by any of the 3 distal impedance channels. It was seen that over $93 \%$ of normal subjects had at least $80 \%$ of liquid swallows, and at least $70 \%$ of viscous swallows, with complete transit (27). Another study in 42 healthy volunteers considered normal a complete bolus clearance in at least $70 \%$ of liquid swallows and $60 \%$ of viscous swallows (28).

A study of 350 patients with various manometric diagnoses found an abnormal transit (considering as normal complete bolus clearance in $80 \%$ of fluid swallows and at least $70 \%$ of viscous swallows) in all patients with achalasia and scleroderma (28). However, most patients with normal manometry, nutcracker esophagus, and isolated LES disorders showed a normal transit. Approximately half of patients with ineffective peristalsis and diffuse esophageal spasm had a normal transit. This study would allow classifying esophageal motor disorders into two groups, with and without impaired esophageal transit (29). However, it seems that monitoring with impedance is more promising in the assessment of uncertain manometric findings such as ineffective peristalsis and diffuse esophageal spasm (DES) (17). In this respect, the current manometric diagnosis to define ineffective peristalsis ( $>30 \%$ of contractions with an amplitude $<30 \mathrm{mmHg}$ ) is highly nonspecific in identifying patients with abnormal transit. One third of patients with ineffective peristalsis have normal fluid and viscous transits, and one third have abnormal fluid and viscous transits (30). Regarding DES, around 25\% of patients have abnormal fluid and viscous transits, and an additional $25 \%$ exhibit abnormal liquid or viscous transits (31). Furthermore, patients with DES, in whom chest pain and high-amplitude contractions predominate, seemingly have a higher percentage of swallows with normal bolus transit as compared to subjects with dysphagia and low-amplitude contractions, who show more esophageal transit impairments. Therefore impedance would seemingly help define different therapy strategies in the various subtypes of patients with DES.
Impedance is of no use in the diagnosis or follow-up of achalasia patients because of its low signal as a result of retained fluid and air trapping in the esophagus $(32,33)$.

While patients with dysphagia have an impaired transit more commonly than patients without dysphagia, the predictive value of impedance is low and its discrimination power is moderate, since there is no clear correlation between transit impairment and perceived dysphagia; hence impedance-manometry would not be superior in all cases to explain symptoms versus manometry alone.

In the research setting new techniques are being developed that incorporate impedance techniques but provide more detail regarding anatomy and mechanical properties of the esophageal body (34).

\section{THE STUDY OF REFLUX DISEASE}

\section{Impedance combined with pH-metry}

\section{Impedance-pH-metry basics and technique}

The principles of impedance combined with $\mathrm{pH}$-metry are those of impedance combined manometry. The catheter consists of a number of cylindrical electrodes $2 \mathrm{~cm}$ apart. Each electrode pair represents a $2-\mathrm{cm}$ segment corresponding to a recording channel. An antimony electrode records $\mathrm{pH}$. The technique is similar to 24-hour ambulatory $\mathrm{pH}$-metry, and a calibration is carried out before catheter insertion using buffer solutions at $\mathrm{pH} 4.0$ and 7.0. Data are recorded around the clock in a portable recorder (35).

Liquid gastroesophageal reflux is detected as an impedance drop that starts at LES and progresses orally. Gas reflux is detected as a simultaneous increase in impedance at two or more impedance segments (Fig. 5).

A detailed consensus nomenclature for reflux patterns obtained with impedance-pH was established (36). Acid reflux is defined when $\mathrm{pH}$ falls below 4 for at least $4 \mathrm{sec}-$ onds or when $\mathrm{pH}$ is below 4 and falls by one unit for at least 4 seconds. Weakly acidic reflux is defined as a $\mathrm{pH}$ decrease by more than one unit for at least 4 seconds when $\mathrm{pH}$ is 4-7. Weakly alkaline reflux is defined when there is evidence of reflux by impedance but $\mathrm{pH}$ does not go down below 7 .

\section{Impedance pH-metry in clinical practice}

Combined monitoring using pH-impedance offers greater sensitivity for the detection of all reflux episodes, and allows us to establish their nature (liquid, gas, mixed), extent (proximal esophagus, pharynx), composition (acidic, weakly acidic, weakly alkaline), and clearance (4).

Impedance monitoring has been validated for acid reflux detection, and identified 97-98\% of acid reflux 


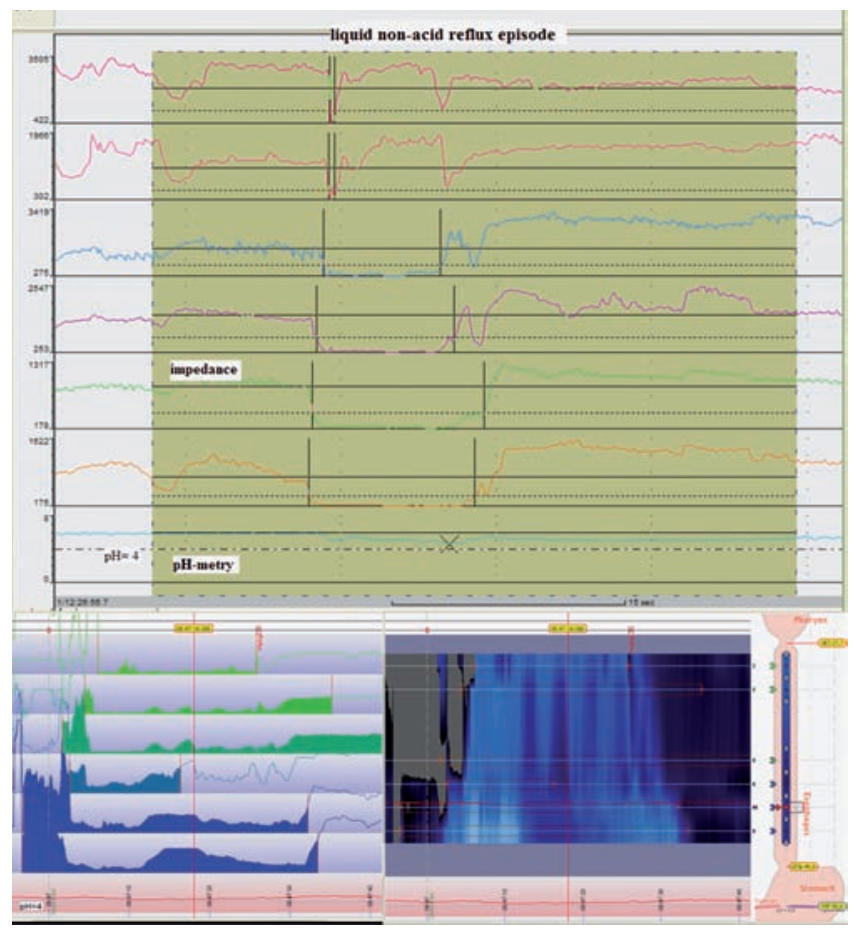

Fig. 5. Impedance-pH. A fluid reflux episode may be seen with a decreased impedance starting at the LES and progressing cephalad. The $\mathrm{pH}$-metry channel shows a $\mathrm{pH}$ fall to just below cutoff $=4$, hence this is a non-acid reflux episode.

episodes recorded with $\mathrm{pH}$-metry in both normal subjects (37) and patients with GERD (38). Most importantly, the technique can detect over $93 \%$ of non-acid reflux events, and acid reflux events added on to reflux undetectable with conventional $\mathrm{pH}$-metry (39).

Reference levels have been established both in adults $(37,40)$ and newborns (41) including the frequency of acid, weakly acidic, and weakly alkaline reflux, as well as the length of exposure to refluxed material at the distal esophagus, and its proximal extent. Parameters obtained using conventional $\mathrm{pH}$-metry are also included in $\mathrm{pH}$-impedance studies. A correlation may also be established between reflux episodes and symptoms, with most commonly used indices including the symptom index and symptom association probability index. This correlation is important as it allows establishing whether symptoms are indeed secondary to reflux, and if negative to investigate other potential causes (42).

Both in patients with GERD and healthy subjects, one third of reflux events are non-acidic, and mixed (fluid and gas) episodes are more common than liquid reflux (4).

A great advantage of $\mathrm{pH}$-impedance in the clinical setting is the study of patients on PPIs in order to identify: a) the adequacy of acid suppression; $b$ ) the association of symptoms with the presence of non-acid reflux; and c) the frequency of reflux episodes. The notion of GERD symptoms refractory to PPIs may be attributed to mildly acidic reflux. A comparison of post-prandial recordings in patients on and off PPI therapy showed a decrease in acid reflux episodes but an increase in non-acid events in patients on PPIs, and that heartburn is replaced by regurgitation (43). Adding impedance to $\mathrm{pH}$-metry improves a study's diagnostic yield by $20 \%$, and allows higher symptom correlation versus $\mathrm{pH}$-metry alone (44).

In patients with PPI-refractory symptoms acid reflux is associated with $7-28 \%$ of persisting symptoms, and weakly acidic reflux with $30-40 \%$ of symptoms; up to $30-60 \%$ of patient-reported symptoms are not associated with any reflux events whatsoever $(43,45)$.

Impedance-pH studies suggest that patients with moderate or severe esophagitis have weakly acidic reflux with a frequency similar to or higher than healthy controls, and that weakly acidic exposure at the distal esophagus is similar for esophagitis and non-erosive GERD, even though a higher number of weakly acidic episodes was seen during the supine period in patients with Barrett's esophagus (46). Patients with esophagitis have more acid reflux than patients without esophagitis, and the weakly acidic reflux pattern is similar in both (47); however, patients without esophagitis are more responsive to weakly acidic reflux, and the presence of gas in the refluxed material increases reflux perception (48). It should be noted that weakly acidic reflux is not synonymous with bile reflux. Bile reflux represents 10-15\% of weakly acidic or weakly alkaline reflux events, and most bile reflux episodes occur concurrently with acid reflux (49).

Another benefit of impedance-pH is the study of atypical reflux symptoms, particularly in respiratory disorders (50). Weakly acidic reflux precedes cough in a subgroup of adult patients with unexplained chronic cough $(51,52)$, and may be highly relevant in patients with lung transplant $(52,53)$.

A higher prevalence of proximal reflux, and the presence of gas in weakly acidic reflux may also explain laryngeal symptoms and pharyngeal globus in patients (54).

To conclude, impedance-pH has allowed establishing that acid reflux is a subtype amongst other potential reflux subtypes, and that most reflux episodes represent mixed episodes consisting of weakly acidic fluid and gas. This type of reflux may explain symptom refractoriness in some patients on PPIs, and in subjects with atypical reflux complaints.

\section{REFERENCES}

1. Ciriza C, García L, Díez A, Delgado M, Fernández AL, Vega A, et al. Role of stationary esophageal manometry in clinical practice. Manometric results in patients with gastroesophageal reflux, dysphagia or non-cardiac chest pain. Rev Esp Enferm Dig 2004; 96: 606-8.

2. Pandolfino JE, Kahrilas PJ. American Gastroenterological Association medical position statement. Clinical use of esophageal manometry. Gastroenterology 2005; 128: 207-8. 
3. Kahrilas PJ, Sifrim D. High-resolution manometry and impedance$\mathrm{pH} /$ manometry: valuable tools in clinical and investigational esophagology. Gastroenterology 2008; 135: 756-69.

4. Sifrim D, Holloway R, Silny J, Xin Z, Tack J, Lerut A, et al. Acid, nonacid, and gas reflux in patients with gastroesophageal reflux disease during ambulatory 24-hour $\mathrm{pH}$-impedance recordings. Gastroenterology 2001; 120: 1588-98.

5. Clouse RE, Staiano A. Topography of the esophageal peristaltic pressure wave. Am J Physiol Gastrointest Liver Physiol 1991; 261: G677-84.

6. Ghosh SK, Pandolfino JE, Zhang Q, Jarosz A, Shah N, Kahrilas PJ. Quantifying esophageal peristalsis with high-resolution manometry: a study of 75 asymptomatic volunteers. Am J Physiol Gastrointest Liver Physiol 2006; 290: G988-97.

7. Fox M, Hebbard G, Janiak P, Brasseur JG, Ghosh S, Thumshirn M, et al. High-resolution manometry predicts the success of oesophageal bolus transport and identifies clinically important abnormalities not detected by conventional manometry. Neurogastroenterol Motil 2004; 16: 533-42.

8. Clouse RE, Staiano A, Alrakawi A, Haroian L. Application of topographical methods to clinical esophageal manometry. Am J Gastroenterol 2000; 95: 2677-9.

9. Pandolfino JE, Ghosh SK, Zhang Q, Jarosz A, Shah N, Kahrilas PJ. Quantifying EGJ morphology and relaxation with high-resolution manometry: a study of 75 asymptomatic volunteers. Am J Physiol Gastrointest Liver Physiol 2006; 290: G1033-40.

10. Pandolfino JE, Kahrilas PJ. New technologies in the gastrointestinal clinic and research: impedance and high-resolution manometry. World J Gastroenterol 2009; 15: 131-8.

11. Ghosh SK, Janiak P, Schwizer W, Hebbard GS, Brasseur JG. Physiology of the esophageal pressure transition zone: separate contraction waves above and below. Am J Physiol Gastrointest Liver Physiol 2006; 290: G568-76.

12. Ghosh SK, Janiak P, Fox M, Schwizer W, Hebbard GS, Brasseur JG. Physiology of the oesophageal transition zone in the presence of chronic bolus retention: studies using concurrent high resolution manometry and digital fluoroscopy. Neurogastroenterol Motil 2008; 20: 750-9.

13. Fox MR, Bredenoord AJ. Oesophageal high-resolution manometry: moving from research into clinical practice. Gut 2008; 57: 405-23.

14. Pandolfino JE, Ghosh SK, Rice J, Clarke JO, Kwiatek MA, Kahrilas PJ. Classifying esophageal motility by pressure topography characteristics: a study of 400 patients and 75 controls. Am J Gastroenterol 2008; 103: 27-37.

15. Ghosh SK, Pandolfino JE, Rice J, Clarke JO, Kwiatek M, Kahrilas PJ. Impaired deglutitive EGJ relaxation in clinical esophageal manometry: a quantitative analysis of 400 patients and 75 controls. Am J Physiol Gastrointest Liver Physiol 2007; 293: G878-85.

16. Nguyen NQ, Tippett M, Smout AJ, Holloway RH. Relationship between pressure wave amplitude and esophageal bolus clearance assessed by combined manometry and multichannel intraluminal impedance measurement. Am J Gastroenterol 2006; 101: 2476-84.

17. Bredenoord AJ, Smout AJ. Esophageal motility testing: impedancebased transit measurement and high-resolution manometry. Gastroenterol Clin North Am 2008; 37: 775-91.

18. Scheffer RC, Samsom M, Haverkamp A, Oors J, Hebbard GS, Gooszen HG. Impaired bolus transit across the esophagogastric junction in postfundoplication dysphagia. Am J Gastroenterol 2005; 100: 1677-84.

19. Bredenoord AJ, Weusten BL, Timmer R, Smout AJ. Intermittent spatial separation of diaphragm and lower esophageal sphincter favors acidic and weakly acidic reflux. Gastroenterology 2006; 130: 334-40.

20. Pandolfino JE, Kwiatek MA, Nealis T, Bulsiewicz W, Post J, Kahrilas PJ. Achalasia: a new clinically relevant classification by high-resolution manometry. Gastroenterology 2008; 135: 1526-33.

21. Imam H, Shay S, Ali A, Baker M. Bolus transit patterns in healthy subjects: a study using simultaneous impedance monitoring, videoesophagram, and esophageal manometry. Am J Physiol Gastrointest Liver Physiol 2005; 288: G1000-6.

22. Fass J, Silny J, Braun J, Heindrichs U, Dreuw B, Schumpelick V, et al. Measuring esophageal motility with a new intraluminal impedance device. First clinical results in reflux patients. Scand J Gastroenterol 1994; 29: 693-702.
23. Srinivasan R, Vela MF, Katz PO, Tutuian R, Castell JA, Castell DO. Esophageal function testing using multichannel intraluminal impedance. Am J Physiol Gastrointest Liver Physiol 2001; 280: G457-62.

24. Nguyen HN, Silny J, Albers D, Roeb E, Gartung C, Rau G, et al. Dynamics of esophageal bolus transport in healthy subjects studied using multiple intraluminal impedancometry. Am J Physiol 1997; 273: G958-64.

25. Blonski W, Hila A, Jain V, Freeman J, Vela M, Castell DO. Impedance manometry with viscous test solution increases detection of esophageal function defects compared to liquid swallows. Scand J Gastroenterol 2007; 42: 917-22.

26. Simren M, Silny J, Holloway R, Tack J, Janssens J, Sifrim D. Relevance of ineffective oesophageal motility during oesophageal acid clearance. Gut 2003; 52: 784-90.

27. Tutuian R, Vela MF, Balaji NS, Wise JL, Murray JA, Peters JH, et al. Esophageal function testing with combined multichannel intraluminal impedance and manometry: multicenter study in healthy volunteers. Clin Gastroenterol Hepatol 2003; 1: 174-82.

28. Nguyen NQ, Rigda R, Tippett M, Conchillo J, Smout AJ, Holloway $\mathrm{RH}$. Assessment of oesophageal motor function using combined perfusion manometry and multi-channel intra-luminal impedance measurement in normal subjects. Neurogastroenterol Motil 2005; 17: $458-65$.

29. Tutuian R, Castell DO. Combined multichannel intraluminal impedance and manometry clarifies esophageal function abnormalities: study in 350 patients. Am J Gastroenterol 2004; 99: 1011-9.

30. Tutuian R, Castell DO. Clarification of the esophageal function defect in patients with manometric ineffective esophageal motility: studies using combined impedance-manometry. Clin Gastroenterol Hepatol 2004; 2: 230-6.

31. Tutuian R, Mainie I, Agrawal A, Gideon RM, Katz PO, Castell DO. Symptom and function heterogenicity among patients with distal esophageal spasm: studies using combined impedance-manometry. Am J Gastroenterol 2006; 101: 464-9.

32. Nguyen HN, Domingues GR, Winograd R, Lammert F, Silny J, Matern S. Impedance characteristics of esophageal motor function in achalasia. Dis Esophagus 2004; 17: 44-50.

33. Conchillo JM, Selimah M, Bredenoord AJ, Samsom M, Smout AJ. Assessment of oesophageal emptying in achalasia patients by intraluminal impedance monitoring. Neurogastroenterol Motil 2006; 18: 971-7.

34. McMahon BP, Frokjaer JB, Liao D, Kunwald P, Drewes AM, Gregersen $\mathrm{H}$. A new technique for evaluating sphincter function in visceral organs: application of the functional lumen imaging probe (FLIP) for the evaluation of the oesophago-gastric junction. Physiol Meas 2005; 26: 823-36.

35. Emerenziani S, Sifrim D. New developments in detection of gastroesophageal reflux. Curr Opin Gastroenterol 2005; 21: 450-3.

36. Sifrim D, Castell D, Dent J, Kahrilas PJ. Gastro-oesophageal reflux monitoring: review and consensus report on detection and definitions of acid, non-acid, and gas reflux. Gut 2004; 53: 1024-31.

37. Shay S, Tutuian R, Sifrim D, Vela M, Wise J, Balaji N, et al. Twenty-four hour ambulatory simultaneous impedance and $\mathrm{pH}$ monitoring: a multicenter report of normal values from 60 healthy volunteers. Am J Gastroenterol 2004; 99: 1037-43.

38. Vela MF. Multichannel intraluminal impedance and $\mathrm{pH}$ monitoring in gastroesophageal reflux disease. Expert Rev Gastroenterol Hepatol 2008; 2: 665-72.

39. Sifrim D, Holloway R, Silny J, Tack J, Lerut A, Janssens J. Composition of the postprandial refluxate in patients with gastroesophageal reflux disease. Am J Gastroenterol 2001; 96: 647-55.

40. Zentilin P, Iiritano E, Dulbecco P, Bilardi C, Savarino E, De Conca $\mathrm{S}$, et al. Normal values of 24-h ambulatory intraluminal impedance combined with $\mathrm{pH}$-metry in subjects eating a Mediterranean diet. Dig Liver Dis 2006; 38: 226-32.

41. Lopez-Alonso M, Moya MJ, Cabo JA, Ribas J, del Carmen Macias $\mathrm{M}$, Silny J, et al. Twenty-four-hour esophageal impedance-pH monitoring in healthy preterm neonates: rate and characteristics of acid, weakly acidic, and weakly alkaline gastroesophageal reflux. Pediatrics 2006; 118: e299-308.

42. de la Morena F, Santander Vaquero C, Cantero J, Perez T, Moreno Otero R. Multichannel intraluminal esophageal impedance: a new frontier in motility. Rev Esp Enferm Dig 2008; 100: 86-9. 
43. Maine I, Tutuian R, Shay S. Acid and non-acid reflux in patients with persistent symptoms despite acid supressive therapy: a multicenter study using combined ambulatory imepdance-pH monitoring. Gut 2006; 55: 1398-1402.

44. Zerbib F, Roman S, Ropert A, des Varannes SB, Pouderoux P, Chaput $\mathrm{U}$, et al. Esophageal $\mathrm{pH}$-impedance monitoring and symptom analysis in GERD: a study in patients off and on therapy. Am J Gastroenterol 2006; 101: 1956-63.

45. Zerbib F, Duriez A, Roman S, Capdepont M, Mion F. Determinants of gastro-oesophageal reflux perception in patients with persistent symptoms despite proton pump inhibitors. Gut 2008; 57: 156-60.

46. Gutschow CA, Bludau M, Vallbohmer D, Schroder W, Bollschweiler E, Holscher AH. NERD, GERD, and Barrett's esophagus: role of acid and non-acid reflux revisited with combined $\mathrm{pH}$-impedance monitoring. Dig Dis Sci 2008; 53: 3076-81.

47. Conchillo JM, Schwartz MP, Selimah M, Samsom M, Sifrim D, Smout AJ. Acid and non-acid reflux patterns in patients with erosive esophagitis and non-erosive reflux disease (NERD): a study using intraluminal impedance monitoring. Dig Dis Sci 2008; 53: 1506-12.

48. Emerenziani S, Sifrim D, Habib FI, Ribolsi M, Guarino MPL, Rizzi $\mathrm{M}$, et al. Presence of gas in the refluxate enhances reflux perception in non-erosive patients with physiological acid exposure of the oe- sophagus. Gut 2008; 57: 443-7.

49. Pace F, Sangaletti O, Pallotta S, Molteni P, Porro GB. Biliary reflux and non-acid reflux are two distinct phenomena: a comparison between 24-hour multichannel intraesophageal impedance and bilirubin monitoring. Scand J Gastroenterol 2007; 42: 1031-9.

50. Bajbouj M, Becker V, Neuber M, Schmid RM, Meining A. Combined $\mathrm{pH}$-metry/impedance monitoring increases the diagnostic yield in patients with atypical gastroesophageal reflux symptoms. Digestion 2007; 76: 223-8.

51. Patterson N, Mainie I, Rafferty G, McGarvey L, Heaney L, Tutuian $\mathrm{R}$, et al. Nonacid reflux episodes reaching the pharynx are important factors associated with cough. J Clin Gastroenterol 2009; 43(5): 4149.

52. Tokayer AZ. Gastroesophageal reflux disease and chronic cough. Lung 2008; 186: S29-34.

53. Blondeau K, Mertens V, Vanaudenaerde BA, Verleden GM, Van Raemdonck DE, Sifrim D, et al. Nocturnal weakly acidic reflux promotes aspiration of bile acids in lung transplant recipients. J Heart Lung Transplant 2009; 28: 141-8.

54. Anandasabapathy S, Jaffin BW. Multichannel intraluminal impedance in the evaluation of patients with persistent globus on proton pump inhibitor therapy. Ann Otol Rhinol Laryngol 2006; 115: 56370 . 